\title{
Stability Analysis of a Prey Refuge Predator-Preymodel with Allee Effects
}

\author{
Unal Ufuktepe ${ }^{1}$, Burcin Kulahcioglu ${ }^{2}$, and Sinan Kapcak ${ }^{1}$ \\ unal.ufuktepe@aum.edu.kw, burcinkulahcioqlu@ieu.edu.tr, Sinan.kapcak@aum.edu.kw \\ ${ }^{1}$ American University of the Middle East Egila-Kuwait, ${ }^{2}$ Izmir University of Economics, Izmir-Turkey
}

We study the impact of the Allee effect and prey refuge on the stability of a discrete time predatorprey model. In this study, we focus on stability behavior of the system with the Allee effect in predator, prey and both populations by using center manifold theorem and type of bifurcations. Based on our analytical and numerical results, we observe that the Allee effects stabilizes the systems dynamics in a moderate value of the prey reguge. 